\title{
Statistical Mechanical Analysis of a Typical Reconstruction Limit of Compressed Sensing
}

\author{
Yoshiyuki Kabashima \\ Department of Computational Intelligence \\ and Systems Science \\ Tokyo Institute of Technology \\ Yokohama 226-8502, Japan \\ Email: kaba@dis.titech.ac.jp
}

\author{
Tadashi Wadayama \\ Department of Computer Science \\ Nagoya Institute of Technology \\ Nagoya 466-8555, Japan \\ Email: wadayama@nitech.ac.jp
}

\author{
Toshiyuki Tanaka \\ Department of Systems Science \\ Kyoto University \\ Kyoto 606-8501, Japan \\ Email: tt@i.kyoto-u.ac.jp
}

\begin{abstract}
We use the replica method of statistical mechanics to examine a typical performance of correctly reconstructing $\mathrm{N}$ dimensional sparse vector $\boldsymbol{x}=\left(x_{i}\right)$ from its linear transformation $\boldsymbol{y}=\boldsymbol{F} \boldsymbol{x}$ of $P$ dimensions on the basis of minimization of the $L_{p}$-norm $\|\boldsymbol{x}\|_{p}=\lim _{\epsilon \rightarrow+0} \sum_{i=1}^{N}\left|x_{i}\right|^{p+\epsilon}$. We characterize the reconstruction performance by the critical relation of the successful reconstruction between the ratio $\alpha=P / N$ and the density $\rho$ of non-zero elements in $\boldsymbol{x}$ in the limit $P, N \rightarrow \infty$ while keeping $\alpha \sim O(1)$ and allowing asymptotically negligible reconstruction errors. We show that the critical relation $\alpha_{c}(\rho)$ holds universally as long as $\boldsymbol{F}^{\mathrm{T}} \boldsymbol{F}$ can be characterized asymptotically by a rotationally invariant random matrix ensemble and $\boldsymbol{F} \boldsymbol{F}^{\mathrm{T}}$ is typically of full rank. This supports the universality of the critical relation observed by Donoho and Tanner (Phil. Trans. R. Soc. A, vol. 367, pp. 4273-4293, 2009; arXiv: 0807.3590) for various ensembles of compression matrices.
\end{abstract}

\section{INTRODUCTION}

Let us suppose a situation that an $N$-dimensional vector $x^{0} \in \mathbb{R}^{N}$ is linearly transformed into a $P$-dimensional vector $\boldsymbol{y} \in \mathbb{R}^{P}$, where $\boldsymbol{y}=\boldsymbol{F} \boldsymbol{x}$, by using a $P \times N$ matrix $\boldsymbol{F} \in \mathbb{R}^{P \times N}$. When $\alpha=P / N<1$, it is generally impossible to reconstruct the original vector $\boldsymbol{x}^{0}$ from $\boldsymbol{y}$ and $\boldsymbol{F}$ correctly. However, if $x^{0}$ is sparse, which means that the fraction $\rho$ of non-zero elements in $x^{0}$ is less than unity, a correct reconstruction may be possible by utilizing prior knowledge of the sparsity. The framework for making efficient use of such a possibility is often termed compressed (compressive) sensing [1]. The research history of this scheme is rather long and dates back to the 1970s [2], [3]. However, since the publication of a series of influential papers in the middle of the 2000s, the horizon of this field has rapidly expanded [4], [5], [6], [7], [8].

For exploiting the sparsity in reconstructing $\boldsymbol{x}^{0}$ from $\boldsymbol{y}$ given $\boldsymbol{F}$, reconstruction schemes based on minimization of the socalled $L_{p}$-norm of $\boldsymbol{x}=\left(x_{i}\right) \in \mathbb{R}^{N}$

$$
\begin{aligned}
& \|\boldsymbol{x}\|_{p}=\lim _{\epsilon \rightarrow+0} \sum_{i=1}^{N}\left|x_{i}\right|^{p+\epsilon} \\
& = \begin{cases}\sum_{i=1}^{N}\left|x_{i}\right|^{p}, & p>0 \\
\text { the number of nonzero elements in } \boldsymbol{x}, & p=0\end{cases}
\end{aligned}
$$

under a linear constraint $\boldsymbol{F} \boldsymbol{x}=\boldsymbol{y}$ have been actively explored. We will hereafter refer to these schemes as $L_{p^{-}}$ reconstruction. In particular, much attention has been paid to $L_{1}$-reconstruction since (1) under the linear constraint can be minimized by using convex optimization methods with a computational cost that is polynomial in $N$ for $p=1$ [9], [10], [11], [12].

The following result on $L_{1}$-reconstruction was reported recenly [6], [8], [13]. Let us suppose that the number of non-zero elements in $\boldsymbol{x}^{0}$ is upper-bounded by $S$. Let us also assume that $\boldsymbol{F}$ is composed of independently and identically distributed (i.i.d.) Gaussian random variables of zero mean and a fixed variance. In addition, we shall consider that the reconstruction is successful if and only if the reconstructed vector $\widehat{x}$ exactly accords to $x^{0}$. Accordingly, if the two inequalities

$$
\begin{aligned}
& \frac{2 S}{N} \ln \left(\frac{N}{2 S}\right)+\frac{2 S}{N}+\frac{1}{N} \ln (2 S) \\
& -\frac{P}{2 N}\left(2^{1 / 4}-1-\sqrt{\frac{2 S}{P}}\right)^{2}<0
\end{aligned}
$$

and

$$
2^{1 / 4}-1-\sqrt{\frac{2 S}{P}}>0
$$

hold simultaneously, the probability of the $L_{1}$-reconstruction failing vanishes as $N$ tends to infinity. The previous studies [14], [15] obtained the critical relations between $\alpha$ and $\rho$ for typical and worst cases when $\boldsymbol{x}^{0}$ is randomly generated under the same assumption of $\boldsymbol{F}$ and for the same criterion on the successful reconstruction as $P, N \rightarrow \infty$ while maintaining $\alpha \sim O(1)$.

The above two results have one thing in common in the sense that the critical relations are evaluated under a success criterion that allows no reconstruction errors. However, in performance evaluations of large systems, it may be more plausible to permit asymptotically negligible errors in characterizing the success of reconstruction. In addition, it may be natural to ask how good a reconstruction can be obtained by using the schemes of $p \neq 1$ and/or other choices of compression matrix $\boldsymbol{F}$.

The purpose of this paper is to answer these questions. More specifically, we utilize the replica method of statistical 
mechanics to explore the typical performance of the $L_{p^{-}}$ reconstruction while allowing asymptotically negligible reconstruction errors in the large system limit for a class of compression matrices [16].

This paper is organized as follows. The next section introduces the model that we will examine. Section III outlines the analysis. In section IV, the results of the analysis are shown in conjunction with an experimental validation. The final section is devoted to a summary.

\section{MODEL DEFINITION}

We will consider the following simple scenario of compressed sensing. Each component of the original vector $\boldsymbol{x}^{0}$, $x_{i}^{0}$, is i.i.d. following a distribution

$$
P(x)=(1-\rho) \delta(x)+\rho r(x)
$$

where $\delta(x)$ denotes Dirac's $\delta$ function and $r(x)$ is an arbitrary distribution with finite first- and second-order moments and without a finite mass at the origin. For simplicity, we assume that the second moment about the origin is unity without loss of generality. Motivated by the observed universality of the critical relation between $\alpha$ and $\rho$ for the successful reconstruction, which was reported by earlier studies [17], [18] for various compression matrices, we characterize $\boldsymbol{F}$ as

$$
\boldsymbol{F}=\boldsymbol{U} \boldsymbol{D} \boldsymbol{V}^{\mathrm{T}}
$$

utilizing a form of singular value decomposition [19], where $\boldsymbol{U}$ and $\boldsymbol{V}$ are samples of uniform distributions over $P \times P$ and $N \times N$ orthogonal matrices, respectively, which are independent of each other and of $\boldsymbol{D}$. T denotes the matrix transpose operation. $D$ is a $P \times N$ diagonal matrix such that the eigenvalues of the $P \times P$ matrix $\boldsymbol{D} \boldsymbol{D}^{\mathrm{T}}$ (or $\boldsymbol{F} \boldsymbol{F}^{\mathrm{T}}$ ) asymptotically follow a certain distribution $f(\lambda)$, the support of which is defined over a bounded interval in $\lambda>0$ as $P=\alpha N$ tends to infinity. The situation in which $\boldsymbol{F}$ is composed of i.i.d. random variables of zero mean and a fixed variance corresponds to the case that $f(\lambda)$ is provided by a distribution of the Marchenko-Pastur type [20]. Let us suppose that data $\boldsymbol{m}^{0}$ (which may represent image, sound, etc.) is expressed as $\boldsymbol{m}^{0}=\boldsymbol{V}^{\mathrm{T}} \boldsymbol{x}^{0}$ by utilizing an orthogonal basis $\boldsymbol{V}^{\mathrm{T}}$ and a sparse coefficient vector $\boldsymbol{x}^{0}$. The assumption of (5) corresponds to a situation in which $x^{0}$ is inferred from projections of $\boldsymbol{m}^{0}$ to randomly chosen $P$ orthogonal directions specified by $\boldsymbol{U}$ in conjunction with certain signal amplification expressed by $D$.

In the following, we will examine the critical relation between $\alpha$ and $\rho$ for correctly reconstructing $x^{0}$ from its compressed expression $\boldsymbol{y}=\boldsymbol{F} \boldsymbol{x}^{0}$ by the $L_{p}$-reconstruction, in particular, for cases of $p=0,1$ and 2. However, such a critical relation disappears when $\boldsymbol{y}$ is smeared by noise of non-negligible strength [21].

\section{OUtLine of RePliCA ANALYSis}

A posterior distribution

$$
P_{\beta}(\boldsymbol{x} \mid \boldsymbol{y})=\frac{e^{-\beta\|\boldsymbol{x}\|_{p}} \delta(\boldsymbol{F} \boldsymbol{x}-\boldsymbol{y})}{Z(\beta ; \boldsymbol{y})}
$$

given a compressed expression $\boldsymbol{y}$ and compression matrix $\boldsymbol{F}$ is the basis of our assessment. The normalization factor $Z(\beta ; \boldsymbol{y})=\int e^{-\beta\|\boldsymbol{x}\|_{p}} \delta(\boldsymbol{F} \boldsymbol{x}-\boldsymbol{y}) d \boldsymbol{x}$ acts as the partition function in statistical mechanics. In the limit $\beta \rightarrow \infty$, the posterior distribution (6) converges to a uniform distribution over solutions of the $L_{p}$-reconstruction which minimize $\|\boldsymbol{x}\|_{p}$ under the constraint $\boldsymbol{F} \boldsymbol{x}=\boldsymbol{y}$. Therefore, one can explore the performance of the $L_{p}$-reconstruction by examining the properties of the posterior distribution (6) in the limit $\beta \rightarrow \infty$.

A distinctive feature of the posterior distribution (6) is that it depends on predetermined (quenched) randomness $\boldsymbol{y}\left(=\boldsymbol{F} \boldsymbol{x}^{0}\right)$ and $\boldsymbol{F}$, which naturally leads us to employ the replica method [22]. More precisely, we evaluate the generating function of the partition function $\Phi(n, \beta)=N^{-1} \ln \left[Z^{n}(\beta ; \boldsymbol{y})\right](n \in \mathbb{R})$ by analytically continuing the functional expressions obtained for $n=1,2, \ldots \in \mathbb{N}$ to $n \in \mathbb{R}$, where $[\cdots]$ denotes averaging with respect to the predetermined random variables $\boldsymbol{y}$ and $\boldsymbol{F}$ (or $\boldsymbol{x}_{0}, \boldsymbol{U}$ and $\boldsymbol{V}$ ). Once the expression of $\Phi(n, \beta)$ is obtained, typical properties of the $L_{p}$-reconstruction can be examined by evaluating the minimized $L_{p}$-norm (per element), $C_{p}$, with the identity $C_{p}=-\lim _{\beta \rightarrow \infty} \lim _{n \rightarrow 0} \beta^{-1}(\partial / \partial n) \Phi(n, \beta)$. Similar schemes have been employed for analyzing various problems concerning information and communication [23], [24] including that of noisy sensing [21].

For $n \in \mathbb{N}$, expanding the $n$-th power of the integral of the partition function yields

$$
\left[Z^{n}(\beta ; \boldsymbol{y})\right]=\int \prod_{a=1}^{n} e^{-\beta\left\|\boldsymbol{x}^{a}\right\|_{p}} \prod_{a=1}^{n} \delta\left(\boldsymbol{F} \boldsymbol{x}^{a}-\boldsymbol{y}\right) \prod_{a=1}^{n} d \boldsymbol{x}^{a} .
$$

Employment of the techniques developed in [25], [26], in conjunction with a heuristic identity $\delta(x)=\lim _{\tau \rightarrow+0}(2 \pi \tau)^{-1 / 2} \exp \left(-x^{2} /(2 \tau)\right)$, leads to

$$
\begin{aligned}
& \lim _{N \rightarrow \infty} \frac{1}{N} \ln \left[\prod_{a=1}^{n} \delta\left(\boldsymbol{F} \boldsymbol{x}^{a}-\boldsymbol{y}\right)\right]_{\boldsymbol{U}, \boldsymbol{V}} \\
& =\lim _{\tau \rightarrow+0}\left\{-\frac{n \alpha}{2} \ln (2 \pi \tau)+\operatorname{Tr} G\left(-\frac{\mathcal{T}(n)}{\tau}\right)\right\} .
\end{aligned}
$$

Here, $[\cdots]_{\boldsymbol{U}, \boldsymbol{V}}$ denotes an average with respect to $\boldsymbol{U}$ and $\boldsymbol{V}$, and $G(x)$ is defined on the basis of the Shannon transform of the eigenvalue distribution $f(\lambda)$, as

$$
\begin{aligned}
G(-x)= & \underset{\Lambda}{\operatorname{extr}}\left\{-\frac{\alpha}{2} \int_{1} f(\lambda) \ln (\Lambda+\lambda) d \lambda+\frac{\Lambda x}{2}\right\} \\
& -\frac{1}{2} \ln x-\frac{1}{2}
\end{aligned}
$$

where $\operatorname{extr}_{X}(\cdots)$ means extremization with respect to $X$. For $x \gg 1$, this function asymptotically behaves as $G(-x) \simeq$ $-(\alpha / 2) \ln x+$ const. irrespectively of $f(\lambda)$ as long as the support of $f(\lambda)$ is defined over a bounded interval in $\lambda>0$. $\mathcal{T}(n)$ is an $n \times n$ matrix whose elements are $T_{a b}=N^{-1}\left(\boldsymbol{x}^{a}-\right.$ $\left.\boldsymbol{x}^{0}\right) \cdot\left(\boldsymbol{x}^{b}-\boldsymbol{x}^{0}\right)$ for $a, b=1,2, \ldots, n$. Under the replica symmetric (RS) ansatz $N^{-1}\left|\boldsymbol{x}^{a}\right|^{2}=Q, N^{-1} \boldsymbol{x}^{a} \cdot \boldsymbol{x}^{b}=q$ and $N^{-1} \boldsymbol{x}^{0} \cdot \boldsymbol{x}^{a}=m$ for $a(\neq b)=1,2, \ldots, n, \mathcal{T}(n)$ has eigenvalues $Q-q-n\left(Q-2 m+Q^{0}\right)$ and $Q-q$ of a single degeneracy and $n-1$ denegeracies, respectively, where 
$Q^{0}=N^{-1}\left|\boldsymbol{x}^{0}\right|^{2}$. These make it possible to define the righthand side of $(8)$ for $n \in \mathbb{R}$. Combining this and the expression for the volume of variables $\left\{\boldsymbol{x}^{a}\right\}$ that satisfy the RS ansatz, which can be assessed by using the saddle-point method and taking an average with respect to $\boldsymbol{x}^{0}$, finally yields

$$
\begin{aligned}
C_{p}= & \operatorname{extr}_{\Theta}\left\{\frac{\alpha(Q-2 m+\rho)}{2 \chi}+\widehat{m} m-\frac{\widehat{Q} Q}{2}+\frac{\widehat{\chi} \chi}{2}\right. \\
& +(1-\rho) \int \phi_{p}(\sqrt{\widehat{\chi}} z ; \widehat{Q}) D z \\
& \left.+\rho \int \phi_{p}\left(\sqrt{\widehat{\chi}+\widehat{m}^{2}} z ; \widehat{Q}\right) D z\right\}
\end{aligned}
$$

where $\Theta=\{Q, \chi, m, \widehat{Q}, \widehat{\chi}, \widehat{m}\}, \chi=\lim _{\beta \rightarrow \infty} \beta(Q-q), D z=$ $\exp \left(-z^{2} / 2\right) d z / \sqrt{2 \pi}$, and

$$
\phi_{p}(h ; \widehat{Q})=\lim _{\epsilon \rightarrow+0}\left\{\min _{x}\left\{\frac{\widehat{Q}}{2} x^{2}-h x+|x|^{p+\epsilon}\right\}\right\} .
$$

We have used the fact $Q^{0} \rightarrow \rho \int r\left(x^{0}\right)\left(x^{0}\right)^{2} d x^{0}=\rho$, which follows from our assumption (4) on the distribution $P(x)$.

At this point, we should note the following issues. Firstly, (10) depends on no properties of $\boldsymbol{D}$ except for the compression rate $\alpha$. This means that the asymptotic performance of the $L_{p}$ reconstruction is unchanged as long as $\boldsymbol{F}^{\mathrm{T}} \boldsymbol{F}$ can be asymptotically characterized by a rotationally invariant matrix ensemble and $\boldsymbol{F} \boldsymbol{F}^{\mathrm{T}}$ is typically of full rank. This is in accordance with the universality on the reconstruction thresholds for various matrix ensembles observed in [17], [18]. Such universality is, however, limited to the noiseless cases; the reconstruction performance does depend on $\boldsymbol{D}$ when non-negligible noises exist [21]. Secondly, $Q$ and $m$ determined by (10) represent values of $N^{-1}\left[|\widehat{\boldsymbol{x}}|^{2}\right]$ and $N^{-1}\left[\boldsymbol{x}^{0} \cdot \widehat{\boldsymbol{x}}\right]$, respectively, as long as the solution of the $L_{p}$-reconstruction $\widehat{\boldsymbol{x}}$ is uniquely determined in typical cases. This means that the typical value of the mean square error (MSE) per element between the original and reconstructed vectors, $\mathcal{E}=N^{-1}\left[\left|\widehat{\boldsymbol{x}}-\boldsymbol{x}^{0}\right|^{2}\right]$, can be assessed as

$$
\mathcal{E}=Q-2 m+\rho
$$

by using the extremum solution of (10). When $\widehat{x}$ typically accords with $\boldsymbol{x}^{0}, Q=m=\rho$ holds, yielding $\mathcal{E}=0$. Therefore, the critical condition between $\alpha$ and $\rho$ for the $L_{p^{-}}$ reconstruction, which can be expressed as the critical rate of the reconstruction limit $\alpha_{c}(\rho)$, can be obtained by examining the stability of the successful solution $Q=m=\rho$ of (10). Thirdly, asymptotically negligible MSEs are allowed for successful reconstruction in the current analysis, whereas no errors are permitted in typical case analyses presented in earlier studies [14], [15]. This means that our assessment is of utility for examining how sensitive the performance is to the criterion of the success of reconstruction. Finally, we must keep in mind that the RS ansatz that is adopted in the current analysis is not necessarily guaranteed to be correct. For instance, the local stability of the RS saddle point is lost
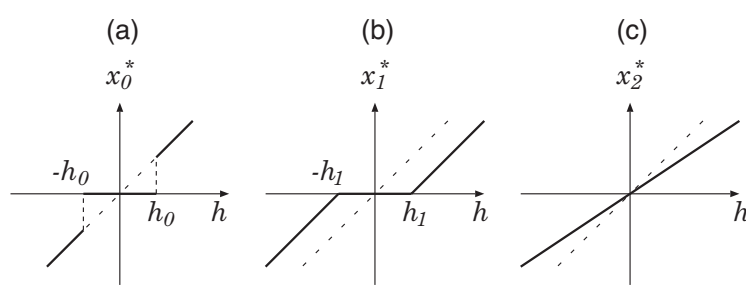

Fig. 1. $x_{p}^{*}(h ; \widehat{Q})$ for $p=0,1$ and 2. (a): $x_{0}^{*}(h ; \widehat{Q})=h / \widehat{Q}$ for $|h|>h_{0}$ and 0 , otherwise, where $h_{0}=\sqrt{2 \widehat{Q}}$. (b): $x_{1}^{*}(h ; \widehat{Q})=(h-h /|h|) / \widehat{Q}$ for $|h|>h_{1}$ and 0 , otherwise where $h_{1}=1$. (c): $x_{2}^{*}(h ; \widehat{Q})=h /(\widehat{Q}+2)$.

against perturbations that break the replica symmetry if

$$
\begin{aligned}
& \frac{\alpha}{\chi^{2}}\left((1-\rho) \int\left(\frac{\partial x_{p}^{*}(\sqrt{\hat{\chi}} z ; \widehat{Q})}{\partial(\sqrt{\widehat{\chi}} z)}\right)^{2} D z\right. \\
& \left.\quad+\rho \int\left(\frac{\partial x_{p}^{*}\left(\sqrt{\widehat{\chi}+\widehat{m}^{2}} z ; \widehat{Q}\right)}{\partial\left(\sqrt{\hat{\chi}+\widehat{m}^{2}} z\right)}\right)^{2} D z\right)>1
\end{aligned}
$$

holds [27]. Here, $x_{p}^{*}(h ; \widehat{Q})=-(\partial / \partial h) \phi_{p}(h ; \widehat{Q})$, the profile of which is shown in Fig. 1 for $p=0,1$ and 2. When the condition (13) holds for the extremum solution of (10), the RS treatment is not valid and one has to explore more general solutions taking into account the effect of replica symmetry breaking (RSB) in order to accurately assess the $L_{p}$-reconstruction.

\section{REsults}

\section{A. Theoretical predictions}

We numerically solved the saddle-point problem (10) for various pairs of $\alpha$ and $\rho$ for $p=0,1$ and 2. When $\rho$ and $p$ are fixed, we always found only the successful solution $Q=m=$ $\rho$ for sufficiently large $\alpha$. As we decreased $\alpha$, the successful solution lost its stability within the RS ansatz and exhibited a transition to a failure solution.

For the successful solution, conjugate variables $\widehat{Q}$ and $\widehat{m}$ are always infinitely large. On the other hand, $\chi$ and $\hat{\chi}$ do not necessarily diverge. We assessed the lower limit of the compression rate for given $\rho, \alpha_{c}(\rho)$, for the $L_{p}$-reconstruction by examining the local stability of the successful solution.

1) $p=0$ : The successful solution is stable if and only if $\alpha>\rho$. This indicates that $\alpha_{c}(\rho)=\rho$ for $p=0$. Even if the positions of the non-zero elements in $\boldsymbol{x}^{0}$ are known, $\alpha>\rho$ is necessary for $\boldsymbol{F} \boldsymbol{x}=\boldsymbol{y}$ to have a unique solution. This implies that the $L_{0}$-reconstruction achieves the best possible performance of compressed sensing. However, the condition (13) always holds for the successful solution because of the discontinuous profile of $x_{p}^{*}(h ; \widehat{Q})$. This means that the assessment under the RS ansatz is not appropriate and further explorations taking RSB into account will be necessary before an accurate performance evaluation of the $L_{0}$-reconstruction can be made within our framework. However, this is beyond the scope of the current study. 
2) $p=1: \hat{\chi}$ of the successful solution is determined by

$$
\begin{aligned}
\hat{\chi}= & \alpha^{-1}\left[2 ( 1 - \rho ) \left((\widehat{\chi}+1) \mathcal{Q}\left(\widehat{\chi}^{-1 / 2}\right)\right.\right. \\
& \left.\left.-\hat{\chi}^{1 / 2} \frac{e^{-1 /(2 \hat{\chi})}}{\sqrt{2 \pi}}\right)+\rho(\widehat{\chi}+1)\right]
\end{aligned}
$$

where $\mathcal{Q}(x)=\int_{x}^{\infty} D t$ is the conventional Q-function. Utilizing the solution of this equation, the stability condition of the successful solution is expressed as

$$
\alpha>2(1-\rho) \mathcal{Q}\left(\widehat{\chi}^{-1 / 2}\right)+\rho
$$

indicating that the reconstruction limit of the $L_{1}$-reconstruction is $\alpha_{c}(\rho)=2(1-\rho) \mathcal{Q}\left(\widehat{\chi}^{-1 / 2}\right)+\rho . \alpha_{c}(\rho)$ also accords with the critical condition of (13), which implies that the RS solution is stable as long as 15 holds. We thus conclude that there is no need of performing the RSB analysis for the performance evaluation of the $L_{1}$-reconstruction.

We should emphasize that the reconstruction limit 15 accords exactly with the limit obtained in [14], [15] under the criterion of a successful reconstruction that permits no reconstruction errors of any order by utilizing techniques of combinatorial geometry. This indicates that the reconstruction limit is not sensitive to the criterion of the successful reconstruction in the sense that it does not change even if we allow asymptotically negligible reconstruction errors as $P=\alpha N \rightarrow \infty$.

Our results also imply that the replica method may be of utility not only to the current problem of compressed sensing but also to the whole field of combinatorial geometry.

3) $p=2$ : The successful solution is stable if and only if $\alpha \geq 1$, which indicates that $\alpha_{c}(\rho)=1$. For $\alpha>\alpha_{c}(\rho)=$ 1 , 133 does not hold and, therefore, there is no need to do the RSB analysis. However, for $\alpha \geq 1$, one can perfectly reconstruct $x^{0}$ by using the Gauss elmination method to solve the linear equation $\boldsymbol{F} \boldsymbol{x}=\boldsymbol{y}$. This leads us to conclude that the $L_{2}$-reconstruction has no capability of compressed sensing.

\section{B. Consideration}

Figure 2 (a) shows $\alpha_{c}(\rho)$ for $p=0,1$ and 2. For comparison, the inset shows plots of (2) and (3) in the limit of $N, P \rightarrow$ $\infty$ while maintaining $\alpha=P / N \sim O(1), \rho=S / N \sim O(1)$. In the case of large systems, the $L_{1}$-reconstruction is performable with a computational cost of $O\left(N^{3}\right)$ by utilizing interior point methods. On the other hand, although the $L_{0}$-reconstruction may potentially give a better reconstruction capability than the $L_{1}$-reconstruction, it is NP-hard. Figure 2 (a) and these indicate that the $L_{1}$-reconstruction is a practical method that possesses both computational feasibility and relatively high reconstruction capability.

There is a huge discrepancy between the typical reconstruction limit obtained here and the one for the worst case of [6], [8], [13], which is shown in the inset. This implies that there may be much room for improvement of the worst-case assessment, although we must keep in mind that the criterion of reconstruction success in the current analysis, which permits asymptotically negligible reconstruction errors as $N \rightarrow \infty$, (a)

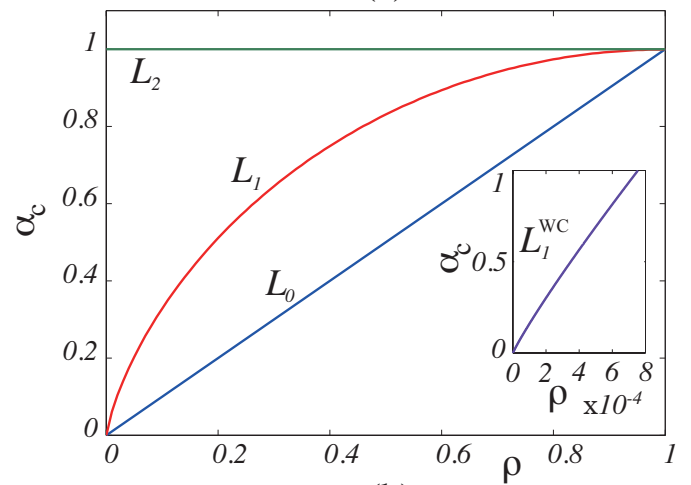

(b)

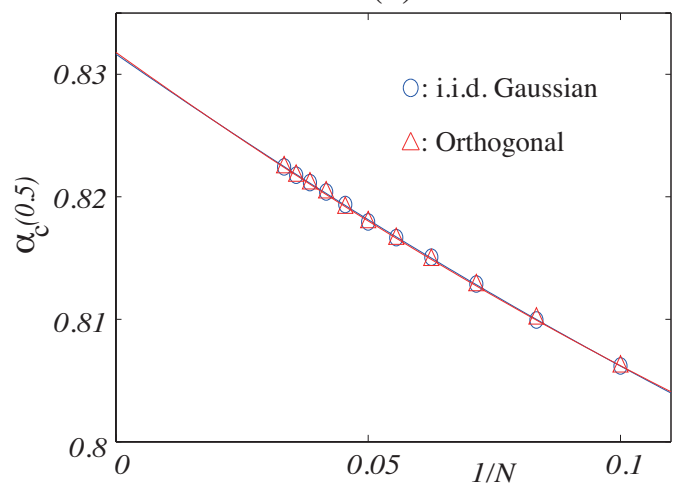

Fig. 2. (a): Typical reconstruction limit for the $L_{p}$-reconstruction of $p=0,1$ and 2. The inset represents the worst case upper-bound for the $L_{1}$-reconstruction offered by 2 and (3). (b): Experimental assessment of $\alpha_{c}(\rho=0.5)$ for the $L_{1}$-reconstruction for (A): matrices of i.i.d. Gaussian random entries (circle) and (B): those of random orthogonal row vectors (triangle). A quadratic fitting with respect to $1 / N$ yields $\alpha_{c}(0.5)=$ $\lim _{N \rightarrow \infty} \alpha_{c}(0.5, N) \simeq 0.83165$ and 0.83179 for $(\mathrm{A})$ and $(\mathrm{B})$, respectively.

is different from that of the worst-case analysis in which no errors are allowed.

\section{Experimental validation}

To justify the result obtained above, we carried out numerical experiments on the $L_{1}$-reconstruction and the original vectors of $\rho=0.5$ for two matrix ensembles; (A): matrices of i.i.d. Gaussian random entries and (B): those of random orthogonal row vectors. The results are summarized in Fig. 2 (b). For both ensembles and each of $N=10,12, \ldots, 30$, we numerically evaluated the critical compression rate for successful reconstruction for $\rho=0.5, \alpha_{c}(\rho=0.5, N)$, on the basis of $10^{6}$ experiments. We employed a package of convex optimization schemes CVX [28], [29] for searching $\widehat{x}$. The critical values of $N \rightarrow \infty$ were estimated by extrapolation of a quadratic fitting with respect to $N^{-1}$, which yielded $\alpha_{c}(0.5)=\lim _{N \rightarrow \infty} \alpha_{c}(0.5, N) \simeq 0.83165$ and 0.83179 for (A) and (B), respectively. These values are in accordance with the theoretical prediction $\alpha_{c}(0.5)=0.83129 \ldots$ up to the third digit (Fig. 2 (b)), which supports the universality of the critical relation. In [6], the critical density $\rho_{c}$ for $\alpha=0.5$ is experimentally evaluated for relatively large systems of 
$N=512$ and 1024 in the case of the i.i.d. Gaussian random entries. Although the exact value is not provided, judging by eye, the plots of the results are close to our theoretical prediction $\rho_{c}(\alpha=0.5)=0.19284 \ldots$. This indicates that our approach at least has the ability to predict these experimental results with high accuracy. However, a general mathematical justification of the replica method is still to be done [30].

\section{SUMmary}

In summary, we assessed a typical reconstruction limit of compressed sensing based on the $L_{p}$-norm reconstruction under linear constraints for $p=0,1$ and 2 in the limit of large systems utilizing the replica method of statistical mechanics. For rotationally-invariant ensembles of the compression matrix $\boldsymbol{F}$, our analysis indicates that the followings hold universally; the replica symmetric solution of the $L_{0}$-reconstruction achieves theoretically optimal reconstruction performance, but unfortunately, is unstable against perturbations that break the replica symmetry, Consequently, further analysis based on the replica symmetry breaking ansatz will be necessary for accurate assessment of its performance. The $L_{2}$-reconstruction has no capability of compressed sensing. On the other hand, the $L_{1}$-reconstruction has good reconstruction ability which is characterized by a universal critical relation between $\alpha$ and $\rho$. Moreover, it is widely known that the $L_{1}$-reconstruction can be done at a computationally feasible cost. These properties point to the great utility of the $L_{1}$-reconstruction in practice.

Our analysis supports that the universality of the critical relation observed in [17], [18] holds for a certain class of the matrix ensembles irrelevantly to the details of the distribution of non-zero elements in the original vector $\boldsymbol{x}^{0}$. However, the relations can depend on the details of $\boldsymbol{F}$ when the ensemble is not rotationally invariant: this situation practically occurs when the original information to be compressed is expanded by non-orthogonal bases and statistically independent sparse coefficients. An analysis on such cases will be reported elsewhere [31].

\section{ACKNOWLEDGEMENT}

This work was partially supported by Grants-in-Aid for Scientific Research on the Priority Areas "Deepening and Expansion of Statistical Mechanical Informatics" from the Ministry of Education, Culture, Sports, Science and Technology, Japan and KAKENHI No. 22300003 from JSPS.

\section{REFERENCES}

[1] E. J. Candès and M. B. Wakin, "An introduction to compressive sampling," IEEE Signal Processing Mag., vol. 25, pp. 21-30, Mar. 2008.

[2] J. F. Claerbout and F. Muir, "Robust modeling with erratic data," Geophysics, vol. 38, pp. 826-844, Oct. 1973.

[3] F. Santosa and W. W. Symes, "Linear inversion of band-limited reflection seismograms," SIAM J. Sci. Stat. Comput., vol. 7, pp. 1307-1330, Oct. 1986.

[4] D. L. Donoho and P. B. Stark, "Uncertainty principles and signal recovery," SIAM J. Appl. Math., vol. 49, pp. 906-931, Jun. 1989.

[5] D. Donoho, "Compressed sensing," IEEE Trans. Inf. Theory, vol. 52, pp. 1289-1306, Apr. 2006.

[6] E. J. Candès and T. Tao, "Decoding by linear programming," IEEE Trans. Inf. Theory, vol. 51, pp. 4203-4215, Dec. 2005.
[7] E. J. Candès, J. Romberg, and T. Tao, "Robust uncertainty principles: Exact signal reconstruction from highly incomplete frequency information," IEEE Trans. Inf. Theory, vol. 52, pp. 489-509, Feb. 2006.

[8] E. J. Candès and T. Tao, "Near-optimal signal recovery from random projections: Universal encoding strategies?" IEEE Trans. Inf. Theory, vol. 52, pp. 5406-5425, Dec. 2006.

[9] E. J. Candès and T. Tao, "The Dantzig selector: Statistical estimation when $p$ is much larger than n," Ann. Statist., vol. 35, pp. 2313-2351, Dec. 2007.

[10] J. Haupt and R. Nowak, "Signal reconstruction from noisy random projections," IEEE Trans. Inf. Theory, vol. 52, pp. 4036-4048, Sep. 2006.

[11] R. Tibshirani, "Regression shrinkage and selection via the Lasso," $J$. Roy. Stat. Soc. Ser. B, vol. 58, pp. 267-288, 1996.

[12] S. S. Chen, D. L. Donoho, and M. A. Saunders, "Atomic decomposition by basis pursuit," SIAM J. Sci. Comput., vol. 20, pp. 33-61, 1998.

[13] E. J. Candès, "The restricted isometry property and its implications for compressed sensing," C. R. Acad. Sci. Paris, Ser. I, vol. 346, pp. 589592, May 2008.

[14] D. L. Donoho, "High-dimensional centrally symmetric polytopes with neighborliness proportional to dimension," Discrete and Computational Geometry, vol. 35, pp. 617-652, May 2006.

[15] D. L. Donoho and J. Tanner, "Counting faces of randomly projected polytopes when the projection radically lowers dimension," J. Amer. Math. Soc., vol. 22, pp. 1-53, 2009.

[16] Y. Kabashima, T. Wadayama, and T. Tanaka, "A typical reconstruction limit for compressed sensing based on $L_{p}$-norm minimization," J. Stat. Mech., L09003 (12pp), 2009.

[17] D. L. Donoho and J. Tanner, "Observed universality of phase transitions in high-dimensional geometry, with implications for modern data analysis and signal processing," Phil. Trans. R. Soc. A, vol. 367, pp. 42734293, 2009

[18] D. L. Donoho and J. Tanner, "Counting the faces of randomly-projected hypercubes and orthants, with applications," to appear in Discrete and Computational Geometry, July 2010; arXiv: 0807.3590.

[19] T. Shinzato and Y. Kabashima, "Perceptron capacity revisited: classification ability for correlated patterns," J. Phys. A: Math. Theor, vol. 41, 324013 (18pp), Aug. 2008.

[20] A. Nica and R. Speicher, Lectures on the Combinatorics of Free Probability Theory, Cambridge Univ. Press, 2006.

[21] S. Rangan, A. K. Fletcher, and V. K. Goyal, "Asymptotic analysis of MAP estimation via the replica method and applications to compressed sensing," Preprint [online] arXiv:0906.3234 2 [cs.IT], 2009.

[22] V. S. Dotsenko, Introduction to the Replica Theory of Disordered Statistical Systems, Cambridge Univ. Press, Cambridge, 2001.

[23] Y. Kabashima and D. Saad, "Statistical mechanics of low-density paritycheck codes," J. Phys. A: Math. Gen., vol. 37, pp. R1-R43, Jan. 2004

[24] T. Tanaka, "A statistical-mechanics approach to large-system analysis of CDMA multiuser detectors," IEEE Trans. Inf. Theory, Vol. 48, no. 11, pp. 2888-2910, Nov. 2002

[25] K. Takeda, S. Uda, and Y. Kabashima, "Analysis of CDMA systems that are characterized by eigenvalue spectrum," Europhys. Lett., vol. 76, pp. 1193-1199, Dec. 2006.

[26] K. Takeda, A. Hatabu, and Y. Kabashima, "Statistical mechanical analysis of the linear vector channel in digital communication," J. Phys. A: Math. Theor, vol. 40, pp. 14085-14098, Nov. 2007.

[27] J. R. L. de Almeida and D. J. Thouless, "Stability of the SherringtonKirkpatrick solution of a spin glass model," J. Phys. A: Math. Gen., vol. 11, pp. 983-990, May 1978.

[28] M. Grant and S. Boyd (2009, Jun.), CVX: Matlab software for disciplined convex programming, [online] Available: http:// stanford.edu/ boyd/cvx

[29] M. C. Grant and S. P. Boyd, "Graph implementations for nonsmooth convex programs," in Recent Advances in Learning and Control (a tribute to M. Vidyasagar) (Lecture Notes in Control and Information Sciences, vol. 95), V. Blondel, S. Boyd, and H. Kimura, Eds. Springer, 2008, pp. 95-110; [online] Available: http://stanford.edu/ boyd/ graph_dcp.html

[30] M. Talagrand, Spin Glasses: A Challenge for Mathematicians: Cavity and Mean Field Models, Springer, Berlin, 2003.

[31] K. Takeda and Y. Kabashima, "Statistical mechanical analysis of compressed sensing utilizing a correlated compression matrix," submitted to ISIT2010, 2010 